\title{
Towards Efficient Use of an Unmanned Aerial Vehicle for Urban Flood Monitoring
}

\author{
Zoe J. Zhu, ${ }^{1}$ Albert Z. Jiang, ${ }^{1}$ Jizhou Lai, ${ }^{2}$ Yang Xiang, ${ }^{1}$ Benjamin Baird ${ }^{1}$ and Edward A. McBean ${ }^{1}$ \\ ${ }^{1}$ University of Guelph, Guelph, Ontario; ${ }^{2}$ Nanjing University of Aeronautics and Astronautics, Nanjing, China.
}

\begin{abstract}
Flooding is a major concern in many cities. It can be devastating, causing considerable property destruction and many fatalities. Real time monitoring of the impact of flooding after precipitation can be crucial in flood control. We study the feasibility of monitoring the spatial extent of flooding using unmanned aerial vehicles (UAVs). Techniques that improve the efficiency of data collection by UAVs are investigated. The results show that a UAV inconjunction with photogrammetry and geographic information systems can provide precise information about potential flooding areas more quickly and more accurately than traditional techniques. This research indicates new directions for research and practice to decrease flooding risks and hazards.
\end{abstract}

\section{Introduction}

\subsection{Urban Flooding and Storm Water Manage- ment}

Urban flooding has increased in recent years (Ashley et al. 2007). It is a concern for both government authorities and individual citizens due to its huge and sometimes devastating impacts on cities and communities affected. Mainland China, for example, experienced many major rainfall events in the summer of 2016. Many cities, including major ones such as Beijing, Shanghai and Guangzhou, have experienced flooding which has threatened the safety, economy and environment of the affected urban areas (Yin et al. 2011). Gathering information on flooding conditions and locations is the first step in developing remedies and prevention plans (Andrade et al. 2013; Casella et al. 2014). However, traditional methods of gathering flooding information rely mainly on field work which can be unsafe, time consuming and ineffective.

To build resilient cities, researchers have employed computer models for predicting flooding and evaluating the effectiveness of response measures. For example, Bambara et al. (2015) proposed a functional method to model scenarios of crises, including flooding, based on risk analysis. However, the method has limited usefulness in real time prediction and crisis management. Due to its limitations, it is most often used for flooding risk assessments but not to monitor real time events.

\subsection{Advantages of Using Airborne Techniques}

Typical airborne techniques (e.g. using airplanes, helicopters or satellites) have the advantage of capturing orthophotos after rainfall events and conjoining local observations to provide a regional perspective. The cost can be high. However, with the in- creasing availability of low cost unmanned aerial vehicles (UAVs), airborne techniques have become popular among researchers. For example, Italian researchers used a low flying UAV to monitor the impact of ocean storms on coastal areas (Casella et al. 2014). Their study provided an understanding of changes in low-lying coasts and their vulnerability to erosion and flooding. Such work highlights the ability to rapidly provide reliable coastal topography data at a relatively low cost. Buckley et al. (2009) suggest that the use of UAVs can provide early detection of flooding conditions in real time.

In this work, we examine the use of UAVs to monitor urban flooding and stormwater management after rainfall events. A UAV was used to collect photos of water impoundments (also referred to as water ponds in this paper) to provide size and location data. By modeling sizes and locations, the performance of urban drainage systems can be observed, and the risk of flooding if there are subsequent rainfalls can be estimated. Observations of ponds on the surface can indicate drainage system deficiencies, improper surface materials, and deformation of the ground. These are all crucial factors to consider when designing urban drainage and stormwater systems.

Figure 1 shows the advantage that a UAV can provide. The three photos show the same geographic location. It is clear that the photo taken by the camera on the UAV (right) has much more up-to-date information when compared with the photo clip taken from Google Maps (left) at the same time. There appears to be a soil stockpile in the photo clip that is no longer present in the photo on the right. The centre photo, from Baidu Maps (the major mapping system used in mainland China), shows structures in the lower part that were completely removed from the site years ago, according to local authorities. This reflects the time advantage of using a UAV, which can be used at any time to acquire

Zhu, Zoe J., Albert Z. Jiang, Jizhou Lai, Yang Xiang, Benjamin Baird and Edward A. McBean. 2017. Towards Efficient Use of an Unmanned Aerial Vehicle for Urban Flood Monitoring. Journal of Water Management Modeling 26:C433. https://doi.org/10.14796/JWMM.C433

(c) CHI 2017. www.chijournal.org ISSN 2292-6062. 
accurate and up-to-date geographical information. Available satellite images from online sources provide outdated depictions of the area. Moreover, this example shows the advantage of a low altitude UAV which produces clearer, more accurate images of topography, and geomorphology more efficiently than traditional satellite imaging systems. It is also noted that UAVs can be deployed to an area quickly and the resulting images are at a high resolution, while satellite methods have a large drawback due to the use of older images and other factors, such as availability, which can impair their accuracy.
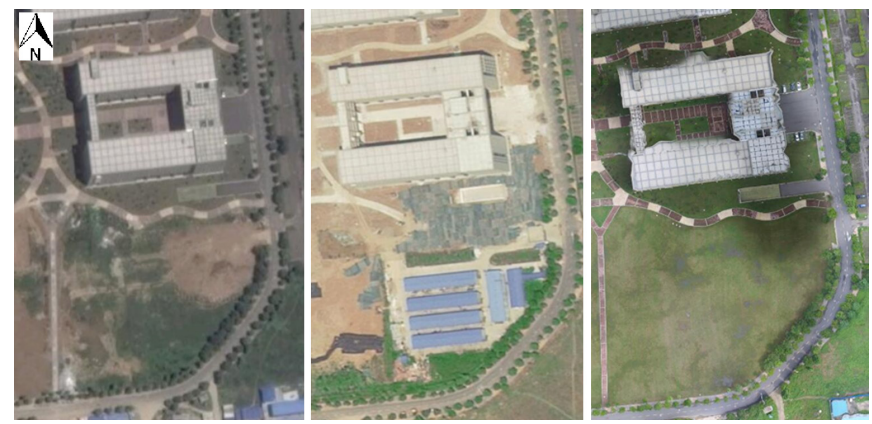

Figure 1 Left: Google map downloaded in June 2016; middle: Baidu map downloaded in June 2016; right: experimental photo taken by the UAV in June 2016 (note: no information was available on the exact date of the satellite image at the time of acquisition).

We used one UAV in our research, with customized flying routes, to monitor the entire region of interest. The UAV took many images with pre-set programs and little or no direct human control. The research objectives were as follows:

to examine the UAV's capability to perform real time monitoring tasks;

to examine various UAV flight plans and thus improve its efficiency by optimising onboard power usage;

to develop an intuitive computer program that will explore various flight plan options; and

to provide information useful for future researches into usse of UAVs for flood monitoring.

\section{Methodology and Results}

\subsection{Flood Monitoring Field Test}

Various factors influence the UAV's effectiveness for flood monitoring. The ones of interest are altitude, velocity and battery power. Altitude and velocity are governed by local air control regulations. Battery power limits the duration and range of the flight. The frequency with which photos are taken and the direction of flight during photo-taking are crucial to the task of observing a given area. In this research, the flying altitude, velocity, photo taking frequency and flight direction were carefully monitored to enable generation of the most efficient flying route(s). The UAV used in this research was the DJI Phantom 3 Standard with all the original accessories, including camera.
The field test consists of the following steps:

1. After a rainfall, the UAV flies over the study area and aerial photos are taken to identify ponding locations. The area of interest was chosen so that it consisted of the typical land cover use of urban areas: medium rise buildings, paved roads and grass fields (Figure 2).

2. The UAV's flying route for the study area was pre-programmed into the vehicle; there was no human interference during the automatic flying procedure.

3. The images taken by the UAV were processed to create a panoramic view of ponding conditions over the entire study area.; this is very useful for both flood management and flood prevention.

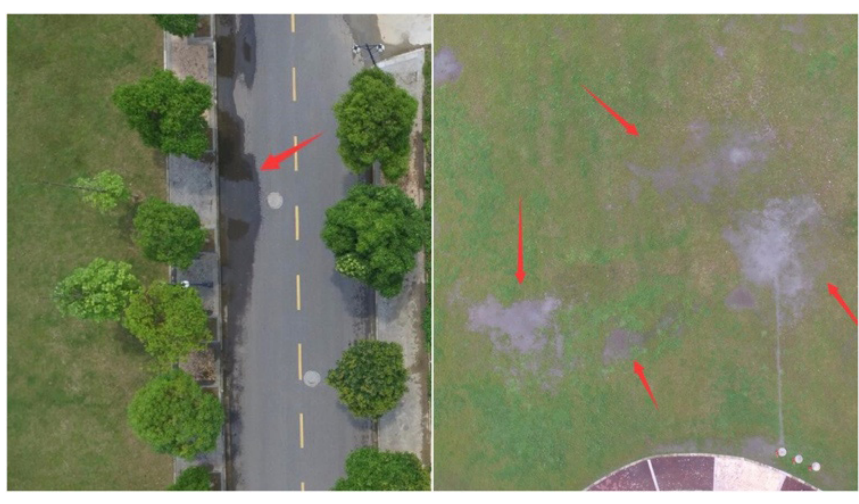

Figure 2 Water ponding on paved road and grassland (arrows indicate ponding).

The appropriate flying altitude for flood monitoring was determined by considering two primary factors: the local air control regulations; and clarity of the photos for later observation. The photos taken by the UAV must be clear enough for a human or computer software to identify impoundment areas and risky flooding locations.

Several experiments were conducted to determine how the areas that the camera can cover will vary at different altitudes. Table 1 shows the results regarding areas covered at different altitudes and the area that can be covered by one single photo taken by the UAV.

Table 1 Flying altitude vs area covered by UAV camera.

\begin{tabular}{cccc}
\hline Flying altitude $(\mathrm{m})$ & Area length $(\mathrm{m})$ & Area width $(\mathrm{m})$ & Total covered area $\left(\mathrm{m}^{2}\right)$ \\
\hline 40 & 53.93 & 71.91 & 3878.24 \\
50 & 66.98 & 89.22 & 5976.02 \\
60 & 79.16 & 105.55 & 8355.65 \\
70 & 93.49 & 124.65 & 11653.66 \\
80 & 106.58 & 142.45 & 15181.61 \\
90 & 120.20 & 160.26 & 19262.51 \\
100 & 136.10 & 181.46 & 24696.89 \\
\hline
\end{tabular}

Areas in Table 1 were calculated by using a reference object with known dimensions in the test area. After a photo was taken at a particular altitude, the object was used as a scale reference to determine the area covered. This was done with a computer that 
processed the pixel information in the picture, including that for the reference object, to determine the total area covered in the photo.

At the maximum experimental flying altitude of $100 \mathrm{~m}$ (the maximum altitude allowed by the local air traffic control), the photo is still sufficiently clear to identify water ponding on various surfaces such as asphalt, pavement and grass fields.

Suitable UAV flying velocity and photo taking frequency were investigated as follows. The UAV was set to fly according to specified GPS waypoints and to take photos automatically. Flying velocity and photo taking frequency are highly interrelated due to the photo overlapping requirements of the processing software, which means the higher velocity will require higher photo taking frequency to ensure that consecutive photos contain overlapped areas. Software packages differ in their requirements for the minimum overlapping areas of consecutive photos in order to perform margin calculations and to stitch them. This process is shown in Figure 3.

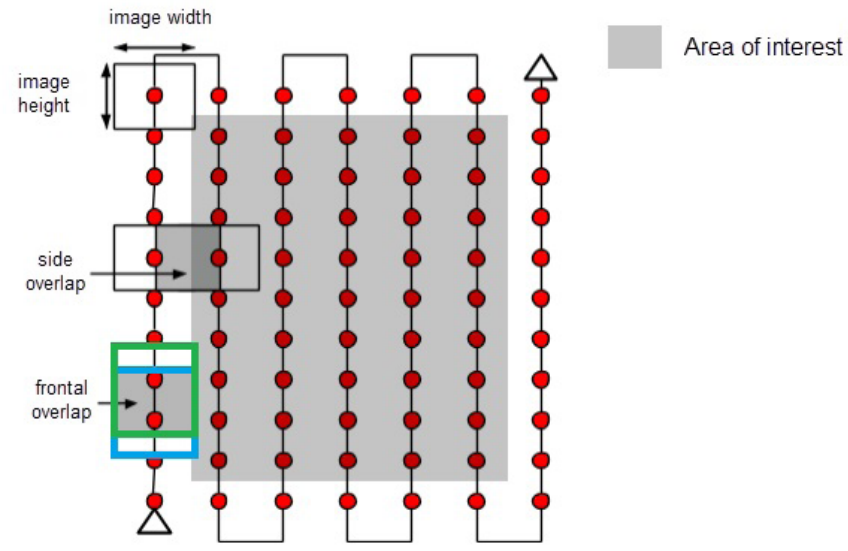

Figure 3 Illustration of photo overlapping (Pix4Dmapper 2016).

In Figure 3, each red dot represents a GPS waypoint where one photo should be taken by the camera on UAV. The black solid lines with arrows at the beginning and end represent the flight route. One rectangle in the upper right of Figure 3 represents the total area that can be covered in one panoramic photo. In order to satisfy the overlapping criteria set by the software, one photo (green) must have a certain overlap area that is covered by the immediately previous photo (blue), as illustrated in Figure 3 (lower left). The side overlap shown in Figure 3 is similarly explained.

PIX4Dmapper, which is photographic panorama processing software for photos taken by UAVs, was selected for use in this study. The areas of frontal overlap (with respect to flight direction) and of side overlap (between flying tracks) were experimentally determined to be $75 \%$ and $60 \%$ for the front and side respectively.

We conducted experiments to verify the software's capability for processing series of photos with various overlapping areas. Table 2 shows the results. In Table 2, Frontal overlap is the percentage of how much area is overlapping in two consecutive photos, where a negative value indicates no overlapping. The Valid? column in Table 2 indicates whether the software can produce the final panorama view (indicated by Yes). As shown by the boldfaced rows (which also show Yes in the Valid? column) in Table 2 , approximately $60 \%$ frontal overlap and $60 \%$ side overlap are sufficient for the software to perform margin calculations and create a panorama, although the recommended values are $75 \%$ and $60 \%$, respectively. For the sake of maximizing battery use, using a $60 \%$ overlapped area is sufficient. However, if the frontal overlap is $<60 \%$, it is almost impossible to produce a panorama view.

Table 2 Straight route velocity and photo test results.

\begin{tabular}{cccccc}
\hline $\begin{array}{c}\text { Altitude } \\
(\mathrm{m})\end{array}$ & $\begin{array}{c}\text { Velocity } \\
(\mathrm{m} / \mathrm{s})\end{array}$ & $\begin{array}{c}\text { Photo interval } \\
(\mathrm{s})\end{array}$ & $\begin{array}{c}\text { Frontal overlap } \\
(\%)\end{array}$ & $\begin{array}{c}\text { Valid? } \\
(\text { Yes/No) }\end{array}$ & $\begin{array}{c}\text { Vertical Length } \\
(\mathrm{m})\end{array}$ \\
\hline 30 & 5 & 5 & 36 & No & 39.18 \\
& 5 & 7 & 11 & No & \\
& 5 & 10 & -28 & No & \\
& $\mathbf{3}$ & $\mathbf{5}$ & $\mathbf{6 2}$ & Yes & \\
40 & $\mathbf{4}$ & $\mathbf{5}$ & $\mathbf{6 3}$ & Yes & 53.93 \\
& 4 & 7 & 48 & No & \\
70 & $\mathbf{5}$ & $\mathbf{7}$ & $\mathbf{6 3}$ & Yes & 93.49 \\
& 5 & 10 & 47 & No & \\
& 5 & $\mathbf{5}$ & $\mathbf{6 3}$ & Yes & 136.10 \\
\hline
\end{tabular}

Although the results from this experiment show that a $60 \%$ frontal overlap is sufficient for creating a panorama, it should not be forgotten that the software specifications indicate that the recommended frontal overlap is $75 \%$. Using a $60 \%$ frontal overlap demonstrates the extreme condition, which pushes to the limit the UAV's maximum flying efficiency.

The commercial UAV used in this experiment has a speed limit of $5 \mathrm{~m} / \mathrm{s}$ in the automatic GPS navigation mode when using its DJI GO application. Options for automatic photo taking intervals are $3 \mathrm{~s}, 5 \mathrm{~s}, 7 \mathrm{~s}$ and $10 \mathrm{~s}$. To maximize battery life, a higher altitude, a faster speed and a larger photo taking interval are preferred. After several limited experiments, we found that 100 $\mathrm{m}$ altitude (maximum allowed by local air traffic control), $5 \mathrm{~m} / \mathrm{s}$ velocity (maximum velocity allowed by the controlling software), and $10 \mathrm{~s}$ photo intervals are the best combination for this typical UAV to satisfy the constraints previously discussed. Using this setting, the UAV completed the task in 7.13 min with a remaining battery power level of $65 \%$. Compared to other flying settings, this combination is optimal.

\subsection{Computational Simulations}

The flying plan we developed is effective for urban flood monitoring in areas where the risk of flooding is uniform across the region. But urban landscapes are not always uniform and can contain tall buildings and large structures, which can adversely affect flood monitoring. To further improve the efficiency of UAV monitoring, the flying plan can specify that the vehicle should avoid flying over large structures and other unrelated areas such 
as undeveloped lands and unoccupied areas. To investigate this potential improvement, we used artificial intelligence (Al) search techniques (Russell and Norvig 2010) to generate more efficient UAV flying plans (shorter flying paths for the same area or covering larger areas without increasing battery usage).

Our computer simulation laid the foundation for the field study to be conducted as future research. The simulation consisted of the following steps. First, the map of the area of interest was converted into a grid net using ArcGIS's fishnet tool, as illustrated in Figure 4. Each cell is labelled, according to the nature of the land, as either to be flown over (e.g. a road or grassland) or not to be flown over (e.g. a large building). Cell size is $120 \mathrm{~m}$ by $160 \mathrm{~m}$, which was chosen based on the results in Table 1 for the UAV at $90 \mathrm{~m}$ (the maximum altitude allowed for UAVs in North America).

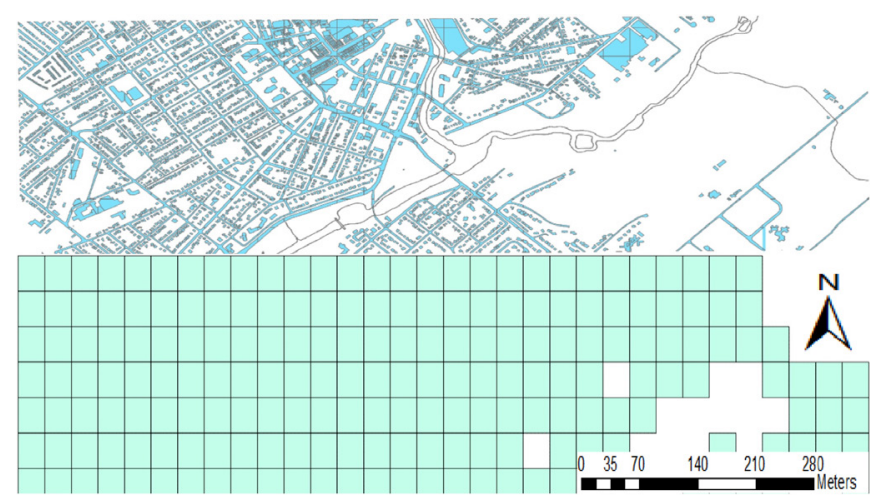

Figure 4 Conversion of ArcGIS map to grid.

The area of interest was represented as a grid. We created a flying plan with the grid as input and with the following properties:

the flying plan starts and ends at specified cells; all cells that are labelled to be flown over are traversed;

cells that are labelled not to be flown over are skipped when possible; and

the total length of the flying path is as short as possible; that is, a cell labelled to be flown over is not traversed more than once if possible.

We developed an algorithm to produce such flying plans. The input to the algorithm includes the dimensions of the grid, the starting cell, the ending cell, and a list of cell blocks that are not to be traversed (e.g. each block that corresponds to a large building).

The output from the algorithm is a flying path which starts at the given starting cell and ends at the ending cell. For each cell and each traverse of the cell, an arrow is used to indicate the direction in which the cell is traversed.

The algorithm has several stages which we describe below. We refer to cells to be flown over as type 1 cells and the remainder as type 0 cells.

The first stage identifies maximal type 1 rectangles in the grid. A type 1 rectangle is a rectangle made of type 1 cells only.
A maximal type 1 rectangle is a type 1 rectangle that cannot be enlarged and remain as a type 1 rectangle.

Figure 5 shows a grid where type 1 cells are in white and type 0 cells are in dark grey. It consists of four maximal type 1 rectangles, as shown in Figure 6. Note that maximal type 1 rectangles are non-overlapping.

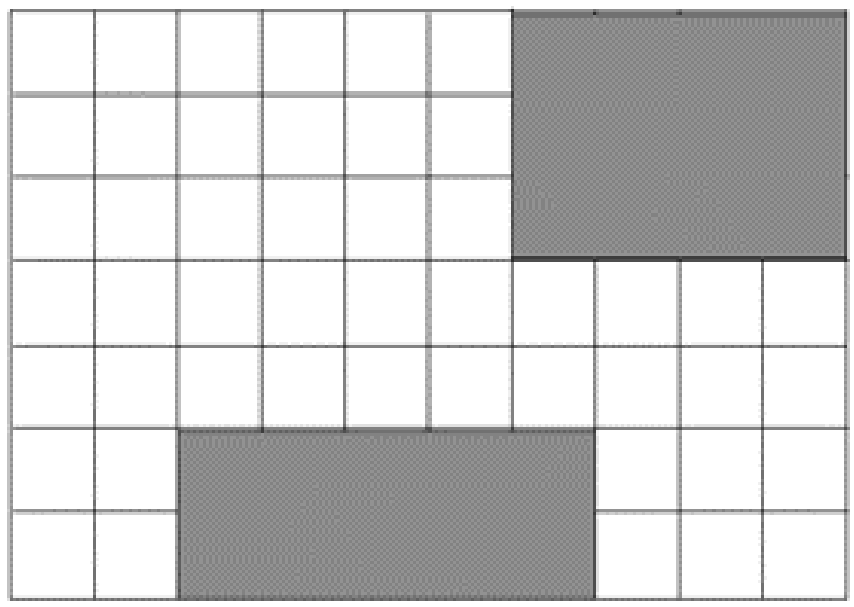

Figure 5 A grid with two building blocks (dark).

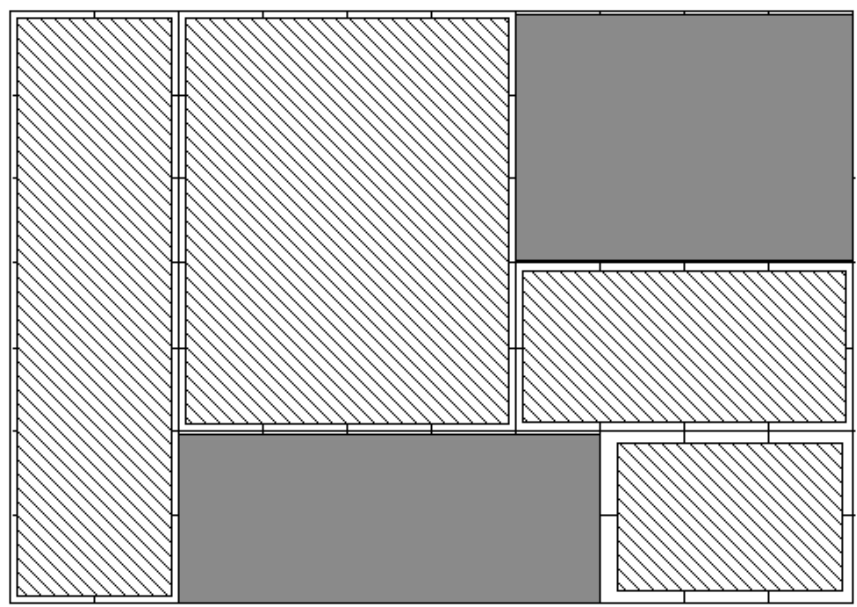

Figure 6 Maximal type 1 rectangles.

A maximal type 1 rectangle can be computed by starting at any type 1 cell, and growing it into a larger rectangle along each side, until no longer possible (i.e. when either type 0 cells or an existing maximal type 1 rectangle is encountered). The maximal type 1 rectangles are not dependent on the starting and ending cells, but are dependent on where the type 0 rectangles are.

The second stage of the algorithm determines the order in which to traverse the maximal type 1 rectangles. The first rectangle to be traversed is the one that is closest to the starting cell or contains it. The last rectangle to be traversed is the one that is closest to the ending cell or contains it. After traversing a rectangle, the closest rectangle is chosen to be the next one to traverse. If there are rectangles at the same minimal distance, the one with the least number of unvisited neighbouring rectangles 
is preferred. If there still is a tie, the smallest rectangle is traversed first.

The third stage of the algorithm is to determine how to traverse any given maximal type 1 rectangle, starting with a given cell within the rectangle. This is done by moving to the closest corner of the rectangle, and then traversing the rectangle either row by row or column by column, depending on which has the longer dimension. For instance, the leftmost rectangle in Figure 6 is traversed column by column.

The fourth stage of the algorithm is to determine how to traverse from a cell $c_{1}$ in the current rectangle to a cell $c_{2}$ in the next rectangle. Cell $c_{1}$ is the last cell visited in the current rectangle. Cell $c_{2}$ is the closest cell to $c_{1}$ in the next rectangle.

The toplevel algorithm GetFlyingPlan combines the above four algorithm stages and generates the flying plan.

GetFlyingPlan identifies all maximal type 1 rectangles in the grid; determines the order to traverse maximal type 1 rectangles; and indexes rectangles as $r_{1}, r_{2}, \ldots, r_{\mathrm{n}}$ according to the order:

$$
s=\text { starting cell; }
$$$$
\text { for } i=1 \text { to } n-1 \text {, }
$$

traverse $r$ starting from cell $s$;

$t=$ last cell visited in ri;

find the cell $z$ in $r_{i+1}$ that is closest to $t$;

traverse from $t$ to $z$;

$s=z$

end for

traverse $r_{n}$ starting from cell $s$;

$t=$ last cell visited in $r_{n^{i}}$

traverse from $t$ to the ending cell;

return the path traversed.

Figure 7 shows a flying plan generated by the algorithm.

The grid is $7 \times 10=70$ cells, where $70-22=48$ cells are type 1 . The flying plan traverses a total of 52 cells, including 4 type 0 cells. In comparison with flying over all cells, it traverses 18 cells less. As indicated in Figure 7, the starting and ending cells of this flying plan are the top left and bottom right cells, respectively. If these cells are altered, a different flying plan will be generated.

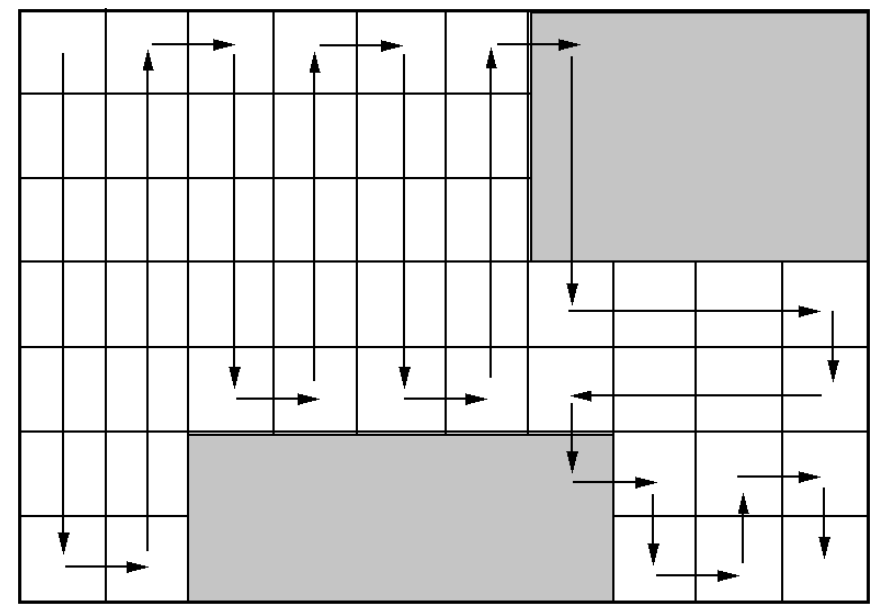

Figure 7 A flying plan generated by the algorithm.
Given an area to be covered, there are generally multiple sets of maximal type 1 rectangles, multiple possible orders to traverse a given set of rectangles, and multiple sequences to traverse cells in a given rectangle. Our algorithm provides a framework to automatically generate a flying plan but the framework does not guarantee that the generated plans are the shortest (optimal). There are many potential optimization techniques that may be applied. One heuristic to find a good order to traverse a given set of rectangles is to visit rectangles according to the distance from their centres to the starting cell (shortest first) or to the ending cell (longest first) or both (order by distance to the starting cell and break ties by distance to the ending cell). Another technique is to guide plan generation by the lower bound of the flying path, which is the total number of type 1 cells. Generate a flying plan and count its length. If the length is close to the lower bound, then it is close to optimal and output the plan. Otherwise, vary the set of rectangles and the traversal order, until a plan that is close to optimal is generated. Our work opens the door to further investigations into optimal plan generation.

In general, as the percentage of type 0 cells increases, that is, as the area has more ground coverage of larger buildings, the flying plan will be more efficient in comparison with traversing the entire area.

A linear path through an $M \times N$ grid, where $M \leq N$, needs $2(M-1)$ changes of direction at the minimum. For instance, the $7 \times 10$ grid in Figure 5 needs at least 12 changes of direction to fly over with a linear path. To avoid flying over certain areas, our algorithm has broken the flying-over area into multiple rectangles, which generally needs more than $2(M-1)$ changes of direction. For instance, the flying plan in Figure 7 avoids flying over the two shaded rectangles, and needs 22 changes of direction.

Although the number of direction changes has increased, their impact is minimal due to the flying physics of drones. Since rotors on a drone are symmetrically located, a change of direction requires only changes of rotation speeds of rotors. No drastic maneuver is required, which is not the case for fixed-wing aircraft. The minimal impact of turns in drones makes a linear flying plan more attractive due to its simplicity in comparison with nonlinear plans.

When the flying plan has been produced, the automatic controller of the UAV is programmed accordingly. The effectiveness of these improved flying plans will be field tested, using the $U A V$, in our future research.

\subsection{Urban Drainage System Modeling}

The urban flood monitoring program is not limited to after-theevent monitoring. It can also be used in the prevention of future urban flooding. One example of this is the use of this method to match observed flooding to existing urban drainage systems or stormwater management systems, which can help to detect issues in existing systems. 
The city water main (red lines) was integrated with the study area (blue shapes indicate all types of structures such as roads or buildings) to create a map, as shown in Figure 8.

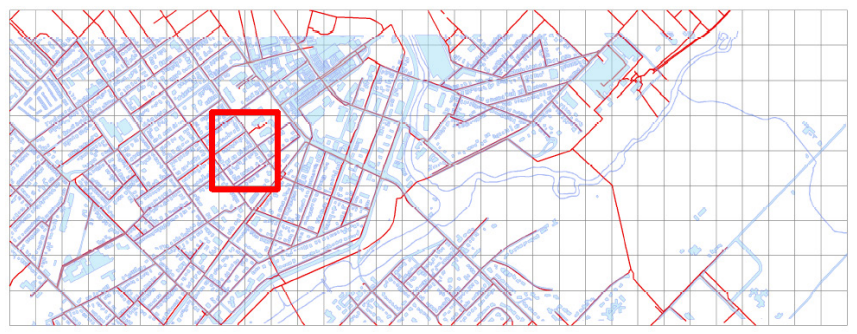

Figure 8 Study area (blue) and storm sewers; the red rectangle indicates four cells zoomed in Figure 9.

A zoomed view of selected four grid cells in Figure 8 is shown in Figure 9 and clearly demonstrates the surface structures and storm sewers.

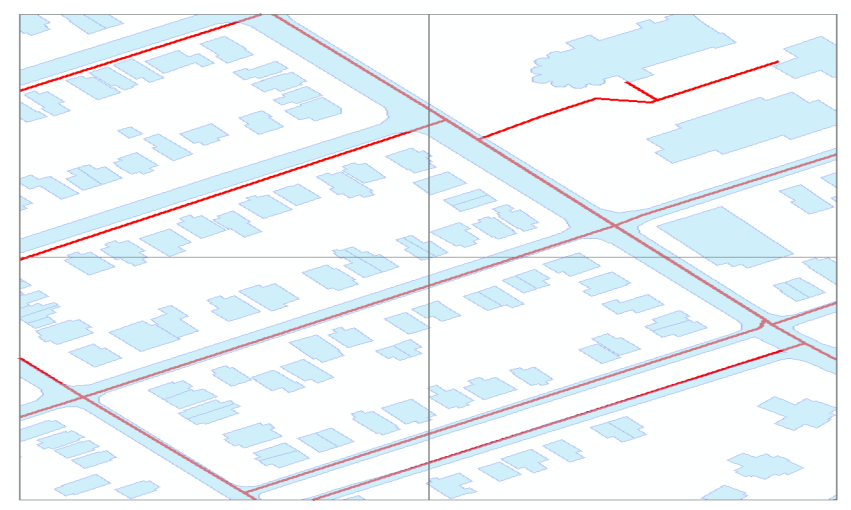

Figure 9 Zoomed view of study area.

This map can be used as a base map for creating the UAV flight plan using the algorithm and method that we described in the previous section. The flying route can be designed to ensure that the UAV coverage includes every cell containing water mains.

Including storm sewers in the creation and analysis of the flying route is only one of many ways in which this UAV technique can be applied to monitor urban flooding and stormwater management related issues. Other examples include the use of land slope, storm water management facilities, lakes and other hydrological features.

\section{Discussion}

The experiments described in the previous section were conducted to explore the effectiveness of using UAV technology in storm water management and modeling. Acquiring the most upto-date information is necessary for creating a better stormwater management monitoring system. The information available in the photos from the single UAV used in this experiment was collected in $<10 \mathrm{~min}$; this data collection would be very time consuming for a person attempting to gather equivalent information in the traditional way. Additionally, many UAVs can be linked to form
UAV networks that can collect data for a larger area in a short time, which is more efficient than it would be if done manually or by using a single UAV. However, we did not test networked UAVs, which will be considered in future studies.

Connecting the single observations to create a view that covers the entire area being studied can provide more information about how the area behaves hydrologically, and how it reacts to a storm event. An area is part of an interconnected system: a flooded street may not only have been caused only by an undersized drainage pipe, for example, but also from runoff contributions due to other causes located some distance away from the flood location. Our research helps to provide a holistic view when monitoring an urban stormwater management system.

The advantages of a UAV over fixed wing planes and helicopters are its low cost and its compact size, which allow it to be deployed more easily than the alternative airborne options. Networks consisting of multiple UAVs, where each UAV has its own coverage area, become very effective for data collection when using the flying route optimization techniques described above. However, security issues have made it harder to obtain a permit for operating a UAV, especially in North America; UAV operation could be delayed if a permit is not obtained in good time. Therefore, for the sake of this research, we wish to introduce this technology and system to municipalities and planning agencies that would benefit from its use. Not only does the UAV need to be improved and adapted to meet our needs, but also the regulations must also be updated. For example, it should be possible for a municipal government to operate such a system with a pre-approved UAV permit, so the delay in obtaining a permit can be avoided and real-time monitoring is easily possible.

There are both limitations in this experiment and room for improvement. The primary limitations are in the equipment and the software. The software used in this experiment requires at least a $75 \%$ frontal overlap for each photo taken by the UAV in order to create the panorama view. Although we reduced this limit to a $60 \%$ frontal overlap and successfully created a panorama view, it should not be forgotten that this overlap amount was designed for the worst case scenario, which required the UAV to cover a large area with its limited battery power. Currently, many UAV mapping applications are available but they all require some frontal and side overlap, and $60 \%$ is a typical value. The limited processing ability of the software has thus significantly hindered the flying efficiency, and must be improved in the future. Another limitation is that each UAV manufacturer uses different software to control the UAV. For example, the DJI Go software used to pilot the UAV in our experiment only has three speed options, which limits our ability to optimize the flying speed.

There are many models of UAV commercially available, as well as custom built UAVs The procedures described in this paper are appropriate for the particular UAV used in the experiment, but some other model will likely have different design parameters and so require different optimal values. However, in such cases, our 
methods can still be used as a guide and the flight plan algorithms are still usable.

\section{Conclusions}

Current urban flooding data that are accurate and rapidly available are valuable for flood evaluation and urban drainage system evaluation and monitoring. We have shown the effectiveness of UAV use in monitoring urban flooding and water ponding conditions by our UAV field tests. We have also developed an algorithm to create efficient UAV flying plans when the urban area contains large buildings. The simulation showed $\sim 30 \%$ area coverage reduction where the density of large structures is moderate, such as on a university campus or in a city centre. As the percentage of large structure coverage in an area increases, the consequent reduction in data gathering area coverage will result in the UAV being able to cover a greater useful area with its limited battery life. The intelligent UAV will also recognize and avoid larger buildings, which increases the safety of UAV use. Our research also showed that city water mains are suitable subjects for the use of UAVs in urban water management. Further optimization and field testing of the algorithm is necessary. For example, the flying route algorithm assumed that starting and ending cells are different. If the drone is required to return to the starting cell after the flight, it could take an optimized path from the ending cell back to the starting cell. That this is a straight line is not included in the generated flying route, but assumed to be the default. Extending the current algorithm so that the returning route is included and the total flight length is minimized will be addressed in future research.

\section{Acknowledgments}

We acknowledge the support of the Ontario-Jiangsu Student Exchange Summer Research Program (http://www.ojs.ouinternational.ca/ and we thank Michael Thai for the programming effort.

\section{References}

Andrade, C. A., Y. F. Thomas, A. N. Lermat, P. Durand and A. Brice. 2013. "Coastal Flooding Hazard Related to Swell Events in Cartagena de Indias, Colombia." Journal of Coastal Research 29 (5): 1126-36.

Ashley, R., J. Blanksby, J. Chapman and A. Zhou. 2007. "Towards Integrated Approaches to Reduced Flood Risk in Urban Areas." In Advances in Urban Flood Management, edited by R. Ashley, S. Gravin, E. Pasche, A. Vassilopoulous and C. Zevenbergen, 415-32. Boca Raton, FL: CRC Press.

Bambara, G., L. Peyra, H. Felix and D. Serre. 2015. “Developing a Functional Model for Cities Impacted by a Natural Hazard: Application to a City Affected by Flooding." Natural Hazards and Earth System Sciences 15:603-15.

Buckley C. D., R. E. Hood and F. J. Lafontaine. 2009. "Application of Airborne Passive Microwave Observations for Monitoring Inland Flooding Caused by Tropical Cyclones." Journal of Atmospheric and Oceanic Technology 26:2051-70.

Casella, L., A. Rovere, A. Pedroncini, L. Mucerino, M. Casella, L. A. Cusati, M. Vacchi, M. Ferrari and M. Firpo. 2014. "Study of Wave Runup Using Numerical Models and Low Altitude Aerial Photogrammetry: A Tool for Coastal Management." Estuarine, Coastal and Shelf Science 149:160-7.

Pix4D. 2016. Pix4Dmapper. Lausanne: Pix4D SA.

Russell, S. and P. Norvig. 2010. Artificial Intelligence: A Modern Approach, 3rd ed. Upper Saddle River, NJ: Prentice-Hall.

Yin, J., Z. Yin, X. Hu, S. Xu, J. Wang, Z. Li., H Zhong and F Gan. 2011. "Multiple Scenario Analyses Forecasting the Confounding Impacts of Sea Level Rise and Tides from Storm Induced Coastal Flooding in the City of Shanghai, China." Environmental Earth Sciences 63:407-14. doi:10.1007/s12665-010-0787-9. 